\title{
Study on the antioxidant ability of procyanidins and their complexes
}

\author{
Di Lu, Lijuan Wang, Wu Zhang, Baohui Guo, and Yuguang Lv* \\ College of Pharmacy, Jiamusi University, Jiamusi, China
}

\begin{abstract}
Due to the excellent antioxidant capacity of proanthocyanidins, combined with the antioxidant mechanism of anthocyanins, it inspired us to study the antioxidant capacity of proanthocyanidins and their complexes. In this article, we conducted antioxidant tests on proanthocyanidin-bovine serum albumin and procyanidin-metal complexes, using DPPH and ABTS methods to determine the antioxidant capacity of procyanidins and their complexes. The results show that compared with proanthocyanidins, the proanthocyanidin complex has a certain degree of improvement in the ability to scavenge free radicals. After being combined with metal ions, the ability to scavenge free radicals is significantly improved. When the ratio of proanthocyanidin $\mathrm{B} 2$ to $\mathrm{Fe}^{3+}$ is $2: 1$, the ability to scavenge free radicals is significantly enhanced.
\end{abstract}

Keyword: Proantho Cyanidins; Anti-oxidation; Free radicals.

\section{Preface}

Proanthocyanidins are a general term for a large class of polyphenolic compounds that are widely present in plants. They have strong antioxidant and free radical scavenging effects, and can effectively eliminate hydroxyl radicals and superoxide anion radicals. They also promote the synthesis of arachidonic acid and phosphoric acid, which protect lipids from peroxidative damage in the process. They are a powerful metal chelator that chelates metal ions to form inert compounds in the body; protects and stabilizes vitamin $\mathrm{C}$ and aids in vitamin $\mathrm{C}$ absorption and utilization.

Oxidative stress is related to the pathogenesis of human degenerative diseases such as diabetes, cancer and cardiovascular disease ${ }^{[1-3]}$. The antioxidant mechanism of anthocyanins is mainly manifested in reducing the accumulation of reactive oxygen species (ROS), scavenging free radicals, and reducing DNA damage.

ROS is an oxygen-containing intermediate metabolite, including oxygen free radicals and non-free radicals. Oxygen free radicals include superoxide anion radicals $\left(\mathrm{O}_{2}{ }^{-}\right)$, three-electron reduction products of oxygen, hydroxyl radicals $(\mathrm{OH})$, peroxy radicals $(\mathrm{ROO})$ and nitric oxide (NO), etc., which are not free radicals. Including the two-electron reduction products of oxygen, hydrogen peroxide $\left(\mathrm{H}_{2} \mathrm{O}_{2}\right)$, peroxynitrite $\left(\mathrm{ONOO}^{-}\right)$, and singlet oxygen $\left({ }^{1} \mathrm{O}_{2}\right)$, etc., which are converted into new free radicals through the oxidation of other components $^{[4-7]}$.

Naturally occurring anthocyanin aglycones and their glycosides have an effect on

*Corresponding author: yuguanglv@163.com 
1,1-diphenyl-2-picrylhydrazyl (DPPH), OH, 2-diazo-bis(3-ethyl) -Benzothiazole-6-sulfonic acid) diammonium salt free radical [2,2'- azinobis (3-ethylbenzothiazoline-6-sulfonic acid ammonium salt), $\mathrm{ABTS}^{+}$], etc., have scavenging ability, and have a slight scavenging ability for $\mathrm{DPPH}$ and $\mathrm{OH}$.Compared with the same concentration of vitamin $\mathrm{C}$ (vitamin $\mathrm{C}, \mathrm{VC}$ ), its ability to clear $\mathrm{ABTS}^{+}$is slightly stronger. ${ }^{[8]}$.

\section{Experimental method}

In this study, two antioxidant detection methods were selected: DPPH method and ABTS method.

\section{Experimental result}

\subsection{Results of the ability of proanthocyanidins to clear DPPH under different pH values}

\subsubsection{The clearance rate of proanthocyanidin samples in absolute ethanol}

According to the sample amount of proanthocyanidin standard solution, the test solutions with mass concentrations of $0.1 \mathrm{mg} / \mathrm{mL}, 0.2 \mathrm{mg} / \mathrm{mL}, 0.3 \mathrm{mg} / \mathrm{mL}, 0.4 \mathrm{mg} / \mathrm{mL}$ and 0.5 $\mathrm{mg} / \mathrm{mL}$ can be obtained. Add sequence according to different samples, the experimental results obtained in parallel 2 groups are: Under the corresponding concentration, the clearance rate of proanthocyanidin in absolute ethanol solution is $91.95 \%, 91.35 \%, 92.80 \%$, $92.65 \%$ and $93.30 \%$.

\subsubsection{Results of the ability of proanthocyanidin samples to remove DPPH under different $\mathrm{pH}$ values}

According to the sample volume of the proanthocyanidin standard solution, the mass concentrations of $0.1 \mathrm{mg} / \mathrm{mL}, 0.2 \mathrm{mg} / \mathrm{mL}, 0.3 \mathrm{mg} / \mathrm{mL}, 0.4 \mathrm{mg} / \mathrm{mL}$ and $0.5 \mathrm{mg} / \mathrm{mL}$ can be obtained at different $\mathrm{pH}$ values. Test the solution, add sequence according to different samples, and the experimental results obtained in parallel for 2 groups are: at the corresponding concentration, the clearance rate of proanthocyanidin absolute ethanol solution after adding different $\mathrm{pH}$ buffer solutions is changed, as shown in Table 1.

Table 1. The clearance rate of proanthocyanidins of various mass concentrations at different $p$ $\mathrm{H}$.

\begin{tabular}{ccccccc}
\hline Concentration & $0.1 \mathrm{mg} / \mathrm{mL}$ & $0.2 \mathrm{mg} / \mathrm{mL}$ & $0.3 \mathrm{mg} / \mathrm{mL}$ & $0.4 \mathrm{mg} / \mathrm{mL}$ & $0.5 \mathrm{mg} / \mathrm{mL}$ & $\mathrm{pH}$ \\
\hline & 95.55 & 95.80 & 95.75 & 95.55 & 95.60 & 3 \\
& 92.90 & 92.45 & 92.85 & 92.55 & 92.95 & 5 \\
Clearance rate & 48.50 & 50.60 & 56.10 & 57.45 & 56.00 & 7 \\
$(\%)$ & 37.45 & 39.50 & 44.25 & 45.05 & 46.80 & 9 \\
& 36.60 & 41.40 & 42.70 & 43.45 & 43.70 & 11 \\
& -16.80 & -14.45 & -14.75 & -14.95 & -13.50 & 13 \\
\hline
\end{tabular}

According to the experimental results, the ability of proanthocyanidins to clear DPPH under different $\mathrm{pH}$ values was drawn as shown in Figure 1.

In the DPPH removal experiment, it can be seen that when proanthocyanidin is under 
acidic conditions, its clearance rate is slightly higher than that of proanthocyanidin ethanol solution. When proanthocyanidin is under neutral and alkaline conditions, its clearance rate is lower than that of proanthocyanidin-ethanol solution. And as the alkalinity increases, the clearance rate decreases.

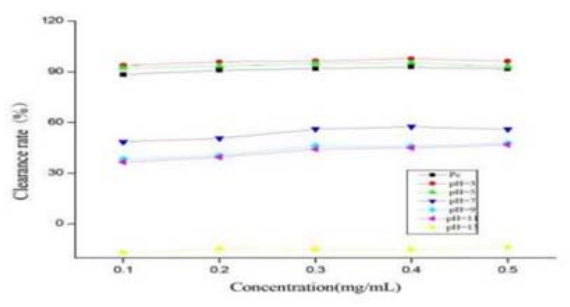

Fig. 1. The ability of proanthocyanidins to clear DPPH under different $\mathrm{pH}$ value.

\subsection{Study on the Antioxidant Activity of Procyanidins in vitro}

\subsubsection{Determination of the ability of proanthocyanidin complexes to scavenge ABTS $^{+}$}

In the experiment, in order to evaluate the anti-oxidation ability of proanthocyanidin complexes, a scavenging experiment was carried out, and the anti-oxidation comparison analysis with Vc was carried out. The experimental results are shown in Figure 2.

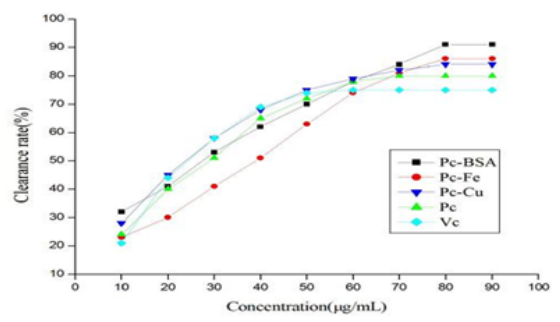

Fig. 2. Proanthocyanidins and its complexes, Vc on the removal of ABTS+.

Figures 2 show that proanthocyanidins, their complexes, and $\mathrm{Vc}$ have a scavenging effect on $\mathrm{ABTS}^{+}$, and the ability to scavenging DPPH gradually becomes stronger as the concentration increases.

Based on the linear regression simulation of procyanidin-iron, proanthocyanidin-copper, proanthocyanidin-bovine serum albumin, proanthocyanidin and $\mathrm{Vc}$ clearance $\mathrm{ABTS}^{+}$antioxidant curve in Figure 2, the correlation coefficient $\mathrm{R}^{2}$ and the linear simulation equation are obtained, and their antioxidant capacity is compared and analyzed.

Table 2. Analysis of anti-oxidation results of proanthocyanidins and their complexes and Vc.

\begin{tabular}{ccc}
\hline sample & Linear regression equation & $\mathrm{R}^{2}$ \\
\hline A: Proantho Cyanidins & $\mathrm{Y}=-0.00126 \mathrm{X}^{2}+1.9547 \mathrm{X}+5.5952$ & 0.9975 \\
B: Procyanidins-Bovine Serum Protein & $\mathrm{Y}=-0.0055 \mathrm{X}^{2}+1.3231 \mathrm{X}+18.143$ & 0.9969 \\
C: Proanthocyanidin-Iron & $\mathrm{Y}=-0.0054 \mathrm{X}^{2}+1.4095 \mathrm{X}+6$ & 0.9876 \\
D: Procyanidin-copper & $\mathrm{Y}=-0.0117 \mathrm{X}^{2}+1.8355 \mathrm{X}+12.238$ & 0.9966 \\
$\mathrm{Vc}$ & $\mathrm{Y}=-0.0159 \mathrm{X}^{2}+2.1737 \mathrm{X}+4.619$ & 0.9749 \\
\hline
\end{tabular}


It can be seen from the analysis of Table 1-2 that the order of $\mathrm{ABTS}^{+}$scavenging ability of the samples is: procyanidin-bovine serum albumin $>$ procyanidin-iron $>$ procyanidin-copper $>$ procyanidin $>\mathrm{Vc}$, and the concentration has a good linear relationship with the clearance rate.

\subsubsection{Determination of the ability of proanthocyanidin complexes to scavenge DPPH}

The scavenging ability of primary free radicals was determined by the DPPH antioxidant activity method. The scavenging ability of proanthocyanidin complex to DPPH was determined, and the results are shown in Figure 3.

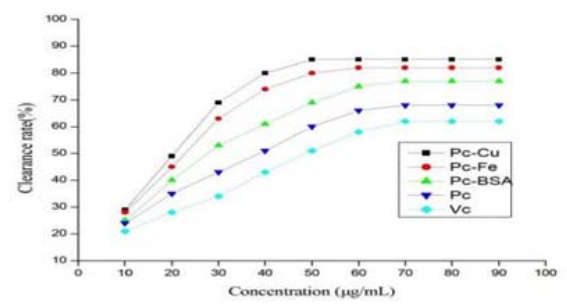

Fig. 3. Proanthocyanidins and its complexes, Vc on the removal of DPPH.

Figure 3 shows that proanthocyanidin and its complexes and $\mathrm{Vc}$ have a scavenging effect on DPPH, and the ability to scavenging DPPH gradually becomes stronger as the concentration increases.

Table 3. Analysis of anti-oxidation results of proanthocyanidins and their complexes and Vc.

\begin{tabular}{ccc}
\hline sample & Linear regression equation & $\mathrm{R}^{2}$ \\
\hline A: Proantho Cyanidins & $\mathrm{Y}=-0.0084 \mathrm{X}^{2}+1.4108 \mathrm{X}+908571$ & 0.9945 \\
B: Procyanidins-Bovine Serum Protein & $\mathrm{Y}=-0.0114 \mathrm{X}^{2}+1.7789 \mathrm{X}+808333$ & 0.9986 \\
C: Proanthocyanidin-Iron & $\mathrm{Y}=-0.0156 \mathrm{X}^{2}+2.1834 \mathrm{X}+8.9524$ & 0.9895 \\
D: Procyanidin-copper & $\mathrm{Y}=-0.0174 \mathrm{X}^{2}+2.3542 \mathrm{X}+9.8095$ & 0.9717 \\
Vc & $\mathrm{Y}=-0.006 \mathrm{X}^{2}+1.158 \mathrm{X}+7.7619$ & 0.9847 \\
\hline
\end{tabular}

It can be seen from the analysis of Tables 3 that the order of the clearance capacity of the samples is: procyanidin-copper>procyanidin-iron>procyanidin-bovine serum albumin $>$ procyanidin $>\mathrm{Vc}$, and the concentration has a good linear relationship with the clearance rate.

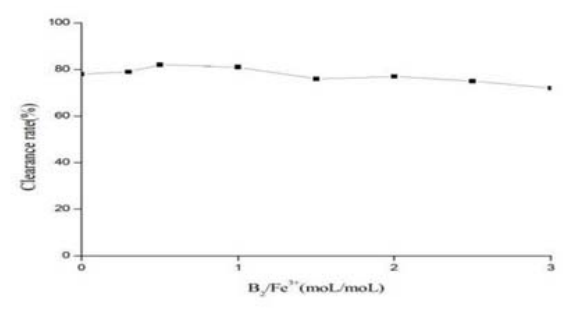

Fig. 4. The scavenging effect of different ratios of proanthocyanidins $\mathrm{B} 2$ and $\mathrm{Fe}^{3+}$ on DPPH free radicals.

It can be seen from Figures 4 that adding a small amount of metal ions to proanthocyanidin B2 has a stronger ability to scavenge free radicals. When the molar ratio 
of proanthocyanidin $\mathrm{B} 2$ to $\mathrm{Fe}^{3+}$ is $2: 1$, the ability to scavenge free radicals is the highest.

\section{Conclusion}

In this subject, the antioxidant capacity of procyanidins and their complexes was determined. The results showed that the antioxidant capacity of procyanidin complexes was improved compared with that of procyanidins. After combining with metal ions, the antioxidant capacity was significantly improved.

\section{References}

1. AZUMA A, YAHUSHIJI H, SATO A. Postharvest light irradiation and appropriate temperature treatment increase anthocyanin accumulation in grape berry skin [J].Postharvest Biol Technol,2019,147(1): 89-99.

2. YOU L, SUI QQ, ZHAO YX, et al. Recent progress in structural modification and physiological activity of anthocyanins [J]. Food Sci.2019,40(11):351-359.

3. ANDRADE TA, HAMERSKI F. LOPEZ FDE, et al. Ultrasound-assisted pressurized liquid extraction of anthocyanins from Aronia melanocarpa pomace [J]. Sep Purif Technol,2021,267(12):119290.

4. KUMAR M, DAHUJA A, SACHDEV A, et al. Evaluation of enzyme and microwave-assisted conditions on extraction of anthocyanins and total phenolics from black soybean (Glycine max L.) seed coat [J]. Int J Biol Macromol,2019,135(8): 1070-1081.

5. FERREIRA FL, MINUZZI NM, RODRIGUES RF, et al. Citric acid water-based solution for blueberry bagasse anthocyanins recovery Optimization and comparisons with microwave-assisted extraction (M AE)[J].LWT,2020,133(11): 110064.

6. ODABAS HI, KOCA I. Simultaneous separation and preliminary purification of anthocyanins from Rosa pimpinellifolia L. fruits by microwave assisted aqueous two-phase extraction [J]. Food Bioprod Process,2021,125(1):170-180.

7. WANG YW, LUAN GX, ZHOU W, et al. Subcritical water extraction, UPLC-Triple-TOF/MS analysis and antioxidant activity of anthocyanins from Lycium ruthenicum Murr [J]. Food Chem,2018,249:119-126.

8. LIMA AS, OLIVEIRA BS, SHABUDIN SV. et al. Purification of anthocyanins from grape pomace by centrifugal partition chromatography[J].J Mol Liq.2021.326(3):115324. 